\title{
The establishment of an optimal migration model for refugees Based on Dijkstra algorithm
}

\author{
Mingyue Sun \\ School of North China Electricity Power University, Baoding 071000, China.
}

\begin{abstract}
Keywords: Dijkstra algorithm, Bayes statistical model, Optimization.
\end{abstract}
\begin{abstract}
In recent years, the problem of large-scale migration of refugees has not only become an international hotspot that has plagued many countries around the world. More than a million migrants and refugees crossed into Europe in 2015, sparking a crisis as countries struggle to cope with the influx, and creating division in the EU over how best to deal with resettling people [1]. Because of the limited choice of refugees, and limited capacity of the recipient countries, the first step is to deal with the allocation of refugees in various routes to different countries. In the second step, a lot of external events will be added to interfere. At this point, it is needed to test whether the model can maintain stability under the interference of certain dynamic factors. Therefore, the basic model needs to meet the conditions of dynamic planning, and Dijkstra algorithm is chosen to optimize the migration route for the refugees. Its advantage is that when the dynamic interference occurs, only some parameters are adjusted slightly, and the basic model is still applicable. Bayes statistical model is used to predict the rate of refugees leaving the transit countries. And the proportion of leaving refugees at each point of action can be obtained. Then, the optimal allocation of the refugees can be calculated based on integer programming.
\end{abstract}

\section{Introduction}

In recent years, the problem of large-scale migration of refugees has not only become an international hotspot that has plagued many countries around the world, but has also become one of the key topics of concern to the 71st Session of the General Assembly. The issue of refugees and immigration concerns world peace and development, affects regional stability and raises a series of political, economic, social and security issues in the face of large-scale refugee and migrants.

The migration of refugees may be a huge burden for the recipient countries. They will consume resources, occupy space, and even because of culture or other reasons conflict with local residents [2]. Therefore, it is a question worth pondering for the European countries that how to plan the whereabouts of the refugees rationally and how to apply the reasonable policy in order to make the refugees and local residents friendly. In this case, the refugees may be a treasure for the receiving country ${ }^{[3]}$.

\section{Optimal Migration Model}

\subsection{Assumptions}

The refugees were at a constant speed when no accident happened.

Infectious diseases will not break out during the process of refugee migration.

The outbreak of conflict between the two sides is only refugees and local residents.

Ignoring the impact of the conflict between the refugees on the risk factor.

\subsection{The Theory and Application of Dijkstra Algorithm}

Dijkstra algorithm is proposed by E.W.Dijkstra in 1959. It applies the greedy algorithm model. And it is currently recognized as the best way to solve the shortest path. The algorithm solves the shortest path problem of a single source point to other vertices in directed graph. The main feature of the algorithm is to select the next vertex at each iteration. The following statement will give a brief introduction to the basic principles. 
Assuming that each node in the network has a label (dt, pt). $\mathrm{dt}$ is the shortest path from the starting point $s$ to $t$. pt is the shortest path from the starting point $s$ to the previous point of $t$. Then dt can be calculated as the following procedure.

1) Initialization of parameters. For the starting point $s$, there is :

Ds $=0$ ps $=$ null

About other points, there is:

$\mathrm{Di}=\infty \mathrm{ps}=$ undersigned

Mark the origin point $\mathrm{s}$. Let $\mathrm{k}=\mathrm{s}$. And all other points are set to not-marked.

2) Check the distance from all labeled point $k$ to other directly connected unlabeled point $j$. And let: $\mathrm{dj}=\min [\mathrm{dj}, \mathrm{dk}+\mathrm{w}(\mathrm{k}, \mathrm{j})]$

And $w(k, j)$ is the length from $k$ to $j$.

3) Select the next point. Select the point $i$ with the shortest length from all the unlabeled point. It is selected as a point contained in the shortest path, and set to be marked.

4) Find the previous point of $i$. Find a point connecting with i directly from all labeled, and mark it as pi .

5) Mark the point $\mathrm{i}$. If all of the point has been marked, and end the algorithm.If not, let $\mathrm{k}=\mathrm{i}$, and return to 2) to continue.

6) The model uses RC to substitute the length of each route, which can get the most secure route. Dijkstra algorithm in dealing with the shortest route problem, the shortest distance between two points is always repressing by the sum of the length of each route. However, in the refugee migration model, directly application of Dijkstra algorithm, the individual results appear RC > 1 of the situation, which the actual does not match. So we have made some changes to the traditional algorithm. Change the formula (8) to the following formula:

$\mathrm{dj}=\min [\mathrm{dj}, 1-(1-\mathrm{dk}) *(1-\mathrm{w}(\mathrm{k}, \mathrm{j}))]$

The final results show as the figure 6 .

\subsection{The theory and application of Bayes Statistical Model}

In probability theory, imagine there is a complete set of events containing $\mathrm{A} 1, \mathrm{~A} 2, \ldots \ldots, \mathrm{Ak}$ that are not compatible with each other. And B is an event with nonzero probability. Then, we have Bayes formula:

$$
P\left(A_{i} \mid B\right)=\frac{P\left(B \mid A_{i}\right] P\left(A_{i}\right)}{\sum_{j=1}^{k} P\left(B \mid A_{i}\right) P\left(A_{i}\right)}, i=1,2, \ldots, k
$$

Now, it will introduce a random variable $\theta$. When event $A j$ appears, it can be defined its value as $\theta_{j}$. Then, the following equation can be obtained.

$\pi\left(\theta_{j}\right)=P\left(\theta=\theta_{j}\right)=P\left(A_{j}\right), j=1,2, \ldots, k$

After further analysis, the following shortened form can be obtained called Bayes statistical model.

$P\left(\theta_{i} \mid x_{1}\right)=\frac{P\left(x_{1} \mid \theta_{i}\right) \pi\left(\theta_{i}\right)}{\sum_{j=1}^{k} P\left(x_{1} \mid \theta_{i}\right) \pi\left(\theta_{i}\right)}, i=1,2, \ldots, k$

In this paper, taking into account the departure rate of the refugees from the transit countries is discrete, computational formula is especially given with discrete parameters and samples as follows.

The prior distribution of the parameters $\theta$ is discrete, and its probability distribution is:

$P\left(\theta=\theta_{i}\right)=\pi\left(\theta_{i}\right), i=1,2, \ldots, k$

And sample $\mathrm{x}=[\mathrm{x} 1, \mathrm{x} 2, \ldots \ldots, \mathrm{xn}] \mathrm{T}$ is also discrete. While $\theta=\theta_{\mathrm{j}}$, its probability distribution is $\mathrm{P}\left(\mathrm{x} \mid \theta_{\mathrm{i}}\right)$. And its posterior distribution is:

$$
\mathrm{P}\left(\theta_{\mathrm{i}} \mid \mathrm{x}\right)=\frac{\mathrm{p}\left(\mathrm{x} \mid \mathrm{\theta}_{\mathrm{i}}\right) \pi\left(\theta_{\mathrm{i}}\right)}{\left.\sum_{\mathrm{j}} \mathrm{P}\left(\mathrm{x} \mid \mathrm{\theta}_{\mathrm{i}}\right) \pi_{(} \mathrm{\theta}_{\mathrm{i}}\right)}, \mathrm{i}=1,2, \ldots, \mathrm{k}
$$




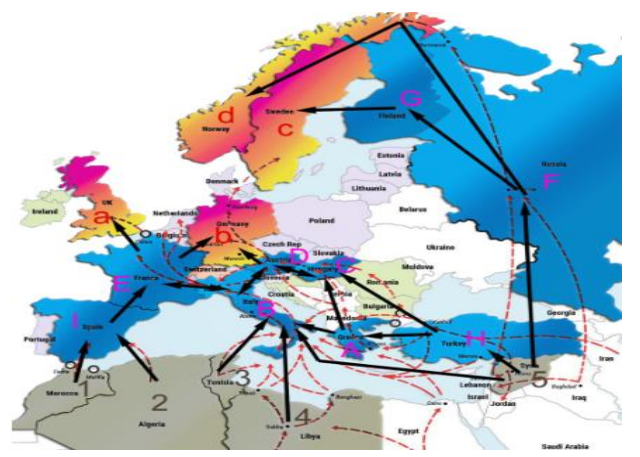

Fig. 1 route sketch map

As is shown in the figure 1, there are five origins, twenty-one routes, nine transit countries and four destinations in this model. The calculation of optimal migration routes achieve two purposes: one is to select a route for refugees from the origin to the destination with the smallest risk coefficient; second, when the number of refugees in a source is excessive, these refugees can be assigned to different routes, to different countries.

We consider comprehensively the number of refugees in each origin, the risk coefficient of each route, economic strength and the maximum number of refugees in every destination. The optimal migration routes can be obtained with above parameters using the Dijkstra algorithm. And the optimal allocation of refugees can be obtained by the integer program.

As mentioned in the hypothesis, the refugees may leave from transit countries at a certain percentage. Therefore, the rate of refugees' departure can be simulated in accordance with the collection of its past data, combining with the Bayes statistical model. Then, the best number of housing in each asylum countries can be determined furtherly.

The figure 2 shows the optimal migration routes between one origin and one destination. As is shown to us, the red line is marked by the refugees from Morocco. Yellow is Algeria, blue is Tunisia, green is Libya and purple is Syria. The proportion of refugees leaving the transit stations can be obtained with the equation (13). As for the leaving refugees, the assignment results as shown below by the integer programming.
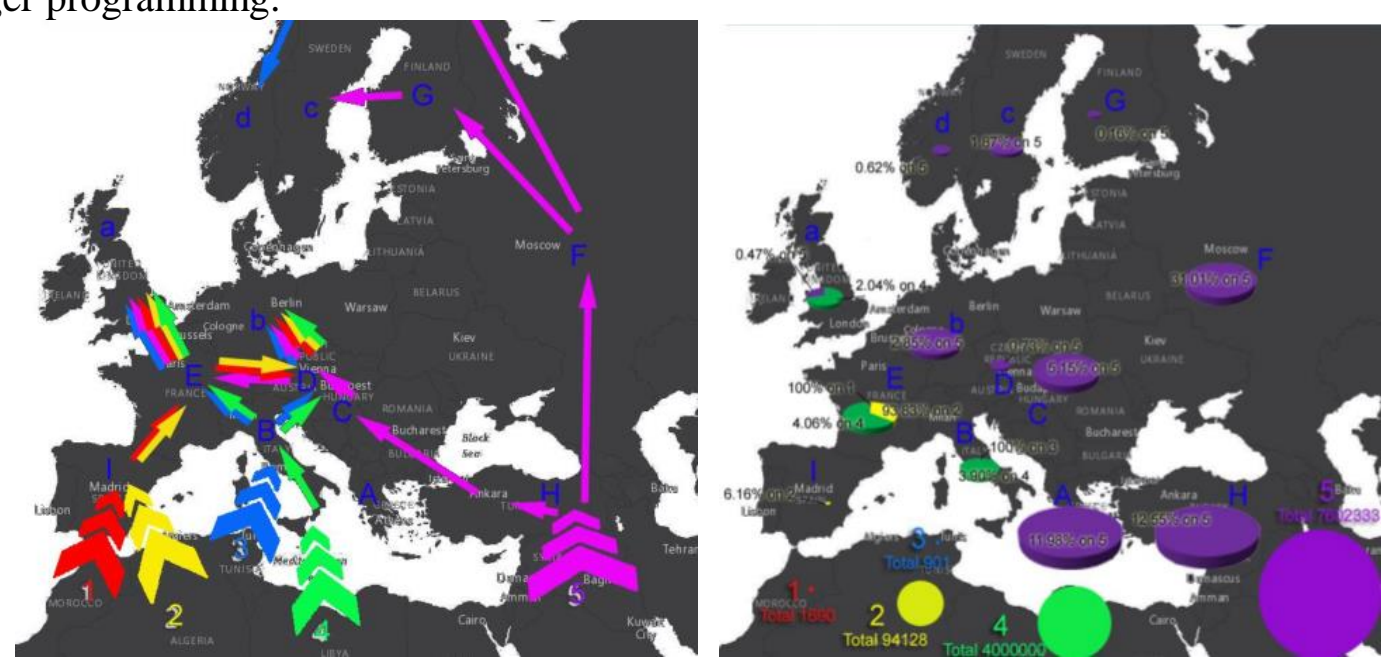

Fig. 2 the optimal migration routes Fig. 3 the final refugee distribution ratio

The proportion of refugees leaving the transit stations can be obtained with the equation (13). As for the leaving refugees, the assignment results as shown below by the integer programming.

The figure 3 shows the final refugee distribution ratio. We can see that the largest number of refugees from Syria. It is the most widely distributed. And the country accounts for the largest proportion of is $\mathrm{H}$ - Turkey. Refugees from Morocco are the least, and are all distributed in France.

\section{Conclusion}


We use the basic migration route model to plan the migration range of 9 stations, 5 stations, 4 destinations and 21 routes. The optimal migration routes between each origin country and each destination country are determined, and the optimal proportion of the refugees in different destinations for each origin are calculated. The key point of the model is the calculation of the risk coefficient. It is an indicator about the degree on danger of each route. And we have chosen the wind, the temperature, distance, GDP, the unemployment rate, the population density and the traffic mode to calculate the risk coefficient.

\section{References}

[1]. Migrant crisis: Migration to Europe explained in graphics http://www.bbc.com/news/world-eur ope-34131911.

[2]. Merle, Kreibaum. Their Suffering, Our Burden? How Congolese Refugees Affect the Ugandan Population [J]. World Development, 2016, 78(1): 262-287.

[3]. Xinhua, net. OECD: refugee is not a burden but a treasure [EB/OL], http://news.ifeng.com/a/20 150927/44746148_0.shtml.

[4]. The truth about asylum - Who's who: Refugee, Asylum Seeker, Refused asylum seeker, Economic migrant, London, England: Refugee Council, retrieved 7 September 2015. 